\title{
CARACTERIZAÇÃO MINERALÓGICA, MORFOLÓGICA E DE SUPERFÍCIE DA ATAPULGITA DE GUADALUPE-PI
}

\author{
K. C. M. Xavier ${ }^{1,2}$, E C. Silva Filho ${ }^{1}$, M. S. F. Santos ${ }^{1}$, M. R. M. C. Santos ${ }^{1}$ e A. B. Luz $^{2}$ \\ 'Laboratório Interdisciplinar de Materiais Avançado - Universidade Federal do Piauí \\ ${ }^{2}$ Ministério da Ciência e Tecnologia e Inovação - MCT, Centro de Tecnologia Mineral - Campus da UESPI \\ edsonfilho@ufpi.edu.br
}

Artigo submetido em outubro/2012 e aceito em outubro/2012

\begin{abstract}
RESUMO
A paligorsquita ou atapulgita $(\mathrm{Mg}, \mathrm{Al})_{2} \mathrm{Si}_{4} \mathrm{O}_{10}(\mathrm{OH}) \cdot 4 \mathrm{H}_{2} \mathrm{O}$ é um argilomineral com morfologia fibrosa, que apresenta uma estrutura cristalina em camada 2:1 com uma dupla camada de tetraedro de silício e uma camada central de octaedro de magnésio, alumínio ou ferro. Os depósitos deste argilomineral ocorrem quase que exclusivamente em solos das zonas áridas e semi-áridas do mundo, sendo que no Brasil, os principais estão localizados em Guadalupe- $\mathrm{Pi}$, e existem várias pesquisas visando ao seu aproveitamento. Este trabalho visa o estudo e caracterização da argila atapulgita do estado do Piauí utilizando as técnicas: difração de Raios $X$ (DRX), fluorescência de raios $X$ (FRX), microscopia eletrônica de
\end{abstract}

varredura (MEV), área superficial especifica (BET), espectroscopia na região do infravermelho com transformata de Fourier (FTIR) e CTC. O resultado da análise de DRX mostrou que esta argila apresenta uma excelente cristalinidade pela intensidade da reflexão $d_{(110)}$. As imagens da microscopia mostraram que a mesma tem uma morfologia fibrosa em forma de bastões ou agulhas. A argila em estudo possui uma área de superfície relativamente alta e capacidade de troca catiônica igual a $40 \mathrm{meq} / 100 \mathrm{~g}$, o que a caracteriza com um potencial adsorvente para adsorção de íons inorgânicos, corantes orgânicos, agentes descorantes, dentre outros.

PALAVRAS-CHAVE: atapulgita, DRX, Área superficial, MEV, FTIR, Guadalupe.

\section{CHARACTERIZATION MINERALOGICAL, MORPHOLOGIC AND SURFACE OF GUADALUPE-PI ATTAPULGITE}

\begin{abstract}
The attapulgite or palygorskite $(\mathrm{Mg}, \mathrm{Al})_{2} \mathrm{Si}_{4} \mathrm{O}_{10}(\mathrm{OH}) \cdot 4 \mathrm{H}_{2} \mathrm{O}$ is a clay mineral with fibrous morphology, which has a crystalline layer with a 2:1 double layer of silicon tetrahedron and a central layer of magnesium, aluminum or iron octahedron. The clay minerals deposits occur almost exclusively in soils of arid and semi-arid regions of the world, and in Brazil, the main are located in Guadalupe-PI, and there are several researches on the use of this clay. This work aims to study the characterization of attapulgite clay from Guadalupe-PI by using the techniques: X-ray diffraction (XRD), X-ray fluorescence (XRF), scanning electron
\end{abstract}

microscopy (SEM), specific surface area (BET), Fouriertransform infrared spectroscopy (FTIR) and CEC. The results of the XRD analysis showed that the clay from Guadalupe has an excellent crystallinity reflection intensity $\mathrm{d}\left({ }_{110)}\right.$. The microscopy images showed that it has a fibrous morphology in the form of rods or needles. The clay under study has a relatively high surface area, and cation exchange capacity of which is $40 \mathrm{meq} / 100 \mathrm{~g}$, which characterizes a potential adsorbent for adsorption of inorganic ions, organic dyes and bleaching agents, among others.

KEY-WORDS: attapulgite, XRD, Surface area, SEM, FTIR, Guadalupe. 


\section{CARACTERIZAÇÃO MINERALÓGICA, MORFOLÓGICA E DE SUPERFÍCIE DA ATAPULGITA DE GUADALUPE-PI}

\section{INTRODUÇÃO}

A atapulgita ou paligorsquita é um argilomineral hidratado de magnésio e alumínio com morfologia microfibrosa, cargas superficiais baixas e alta área superficial (Neaman \& Singer, 2004). Segundo Garcia-Romero, et al. (2004), a estrutura da atapulgita é do tipo 2:1 com duas folhas de tetraedros de òxidos de silicio unidas por uma folha central de octaedros de óxidos de magnésio e alumínio. Estão presentes na folha octaédrica os cátions $\mathrm{Mg}$ (II) e $\mathrm{Al}$ (III), podendo o átomo de alumínio sofrer substituição isomórfica pelos cátions $\mathrm{Fe}(\mathrm{II}), \mathrm{Fe}(\mathrm{III})$, Ti (IV) , Mn (IV) ou ficar vago (A. Mifsud, et al., 1978).

Bradley (1940) propôs o primeiro padrão estrutural para a atapulgita e sugeriu que o argilomineral possue a fórmula [(Mg,Al) $\left.)_{2} \mathrm{Si}_{4} \mathrm{O}_{10}(\mathrm{OH}) .4 \mathrm{H}_{2} \mathrm{O}\right]$ (Corma, et al., 1987; Gonzalez, et al., 1989a; Gonzalez, et al., 1989b). Esta fórmula mostra que o argilomineral contém três formas de água na sua estrutura: (a) coordenada a cátions da folha octaédrica, (b) zeólitica presente nos canais no qual interage tanto com a molécula de $\mathrm{H}_{2} \mathrm{O}$ coordenada quanto a folha tetraédrica e (c) água hidroxila ligada à estrutura do argilomineral no centro da folha octaedrica de acordo Gionis, et al. (2006).

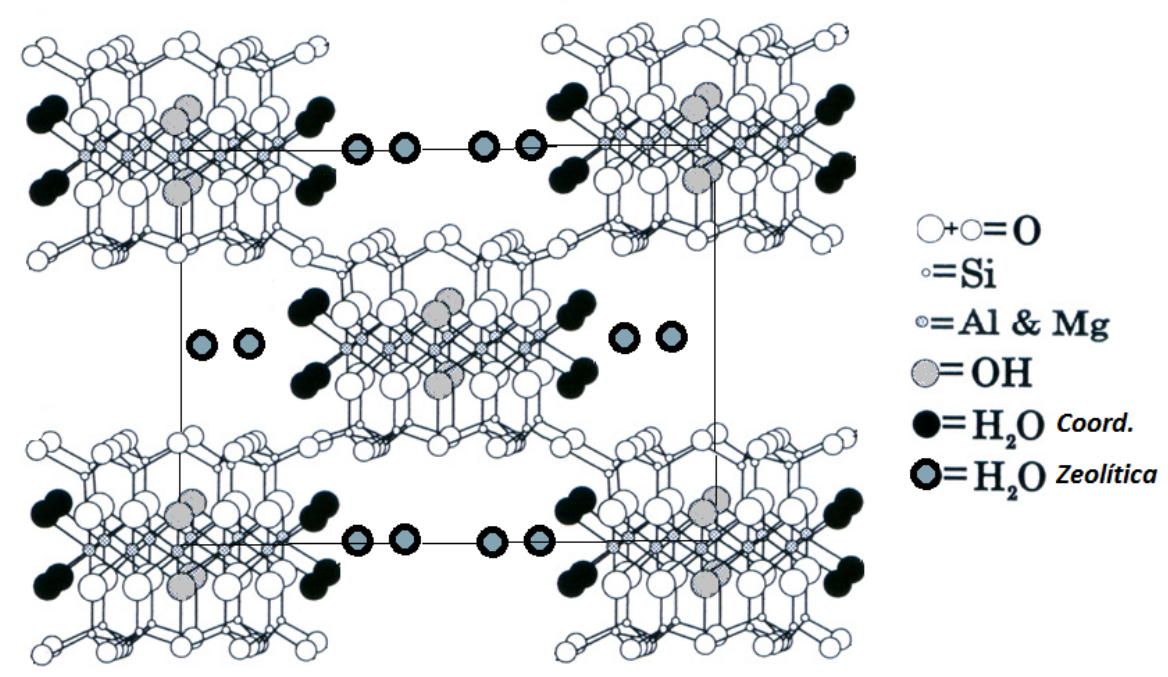

Figura 1 - Detalhes da estrutura cristalina da atapulgita.

A atapulgita ocorre geralmente em solos das zonas semiárida e áridas do mundo (Neaman \& Singer (2004). De acordo com Luz \& Almeida (2005), os principais depósitos de atapulgita no Brasil estão situados no município de Guadalupe-PI localizado a cerca de $300 \mathrm{Km}$ da capital do Estado, conforme o Mapa ilustrativo apresentado na Figura 2, distribuídos por uma área de cerca de $700 \mathrm{~km}^{2}$, onde foram realizados vários trabalhos de pesquisa visando ao desenvolvimento e o aproveitamento deste argilomineral para diferentes usos industriais (Baltar, et al., 2009; Luz \& Almeida, 2005; Luz, et al., 1988; Neto, et al., 1993). 
Com base nas propriedades deste argilomineral exitem numerosas aplicações industriais tais como: lamas de perfuração, adsorventes industriais, adsorventes de cama de gato, suportes de catalisadores, agentes gelificante e descorantes de óleos (Álvarez-Ayuso \& García-Sánchez, 2007; Baltar, et al., 2009; Lei \& Wen, 2008; Li, et al., 2012; Liu, et al., 2008; Stathatos, et al., 2012; Wang, et al., 2012).

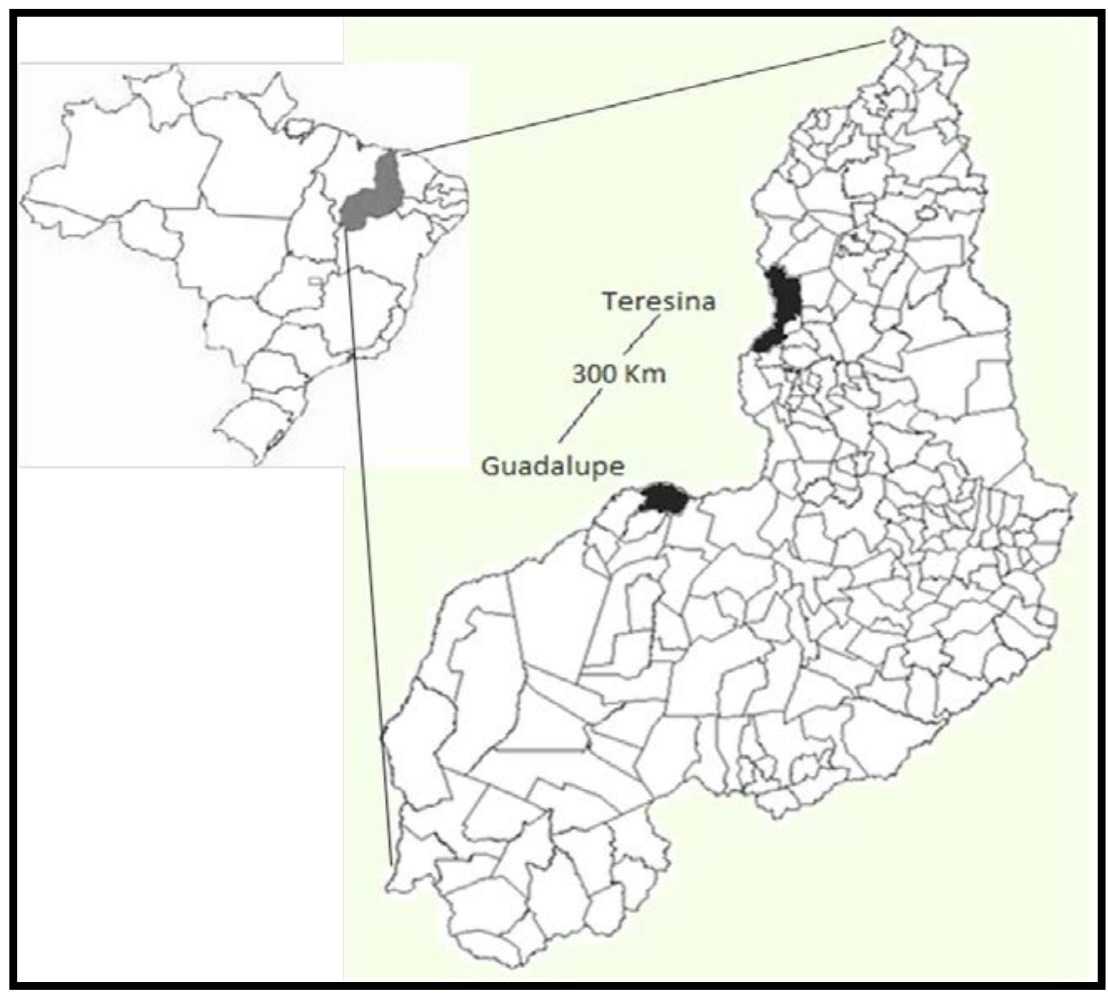

Figura 2 - Mapa de localização do município de Guadalupe-PI.

É objetivo deste estudo caracterizar a atapulgita de Guadalupe - PI, avaliando as suas propriedadedas mineralógicas, morfológica, estrutural, espectroscópica e de superficie por meio das técnicas de DRX, MEV, FRX, FTIR, BET e CTC e compará-los com dados da literatura.

\section{METODOLOGIA}

\section{Processamento da atapulgita}

A amostra da argila atapulgita foi extraída de uma jazida localizada no município de Guadalupe - PI, fornecida pela empresa Itauoeste. A atapulgita natural foi submetida a um processo de homogeneização/quarteamento para obtenção de uma amostra mais homogênea. A desagregação foi efetuada com o auxilio de moinho do tipo martelo e macerada em pistilo e almofariz de porcelana, seguido de peneiramento em malhas de 200 mesh e secagem a $70 \pm 10^{\circ} \mathrm{C}$ em estufa, por 24 horas, para eliminar o excesso de umidade no material. 


\section{Caracterização da atapulgita}

- Difração de raios $X(D R X)$

A amostra de atapulgita natural foi caracterizada por Difração de Raios X (DRX), em um equipamento Labx - XRD 600, da Shimadzu (LIMAV/UFPI), com radiação Cu-K $\alpha(\lambda=1,5406 \AA$ ) com $2 \theta$ no intervalo entre $5^{\circ}$ a $75^{\circ}$, com taxa de varredura de $2^{\circ} / \mathrm{min}$ e tempo total de exposição de 40 minutos.

- Fluorescência de raios X (FRX)

A análise de FRX foi realizada para determinar os principais constituintes minerais presente na amostra de atapulgita. Os teores dos principais óxidos presentes neste argilomineral foram determinados por análise semiquantitativa em espectrômetro por fluorescência de raios X - (WDS), modelo BOL - FRX - 030 (CETEM-RJ). A amostra foi preparada por fusão na diluição de 1 / 10 e como fundente usou-se uma mistura de boratos de lítio $\left(\mathrm{Li}_{2} \mathrm{~B}_{4} \mathrm{O}_{7}-\mathrm{LiBO}_{2}\right)$. Os resultados estão expressos em porcentagem (\%), calculados como óxidos e normalizados a $100 \%$.

- Espectroscopia no Infravermelho com Transformata de Fourier (FTIR)

A espectroscopia de Infravermelho foi realizada para elucidar a caracterização por intermédio das energias vibracionais das ligações metal-ligante presentes na argila. A análise foi realizada usando pastilhas de $\mathrm{KBr}$ (na região de 4000 até $400 \mathrm{~cm}^{-1}$ ) em espectrômetro Varian, 660 - IR (LIMAV/UFPI), um total de 96 varreduras e resolução de $4 \mathrm{~cm}^{-1}$ onde foram utilizadas para obter uma boa relação sinal-ruído.

- Microscopia Eletrônica de Varredura (MEV)

A microscopia eletrônica de varredura (MEV) foi realizada para determinar a morfologia superficial da argila. As micrografias foram obtidas em microscópio eletrônico de varredura após a metalização das amostras com ouro e paládio usando o microscópico JEOL T300 (IQ-UNICAMP).

- Área superficial Especifica (BET)

A área superficial foi determinada na amostra de argila natural, em equipamento de adsorção física de nitrogênio Quantachrome NOVA 4200 (IQ-UNICAMP).

- Capacidade de troca catiônica (CTC)

A capacidade de troca catiônica da atapulgita natural foi determinada pelo metodo azul de metileno seguindo a metodologia da literatura de Morales-Carrera, et al. (2009), devido ser método fácil tem sido bastante utilizado em solos e argilas. 
Análise de DRX

A Figura 1 mostra o difratograma de raios-X da atapulgita natural, onde as principais reflexões referentes à atapulgita e quartzo foram identificadas com o auxilio das fichas cristalográficas dos cartões JCPDF de $\mathrm{n}^{\circ} 31-0783$ e 1-0850794, respectivamente. A região referente à reflexão de maior intensidade da paligorsquita $d_{(110)}$ está localizada aproximadamente em $8,50^{\circ}$. Outras reflexões características deste argilomineral presentes no difratograma e de menor intensidade, também foram identificadas como a $d_{(200)}$ em $14,00^{\circ}$ e $\mathrm{d}_{(400)}$ em $28,10^{\circ}$, bem como a região próxima em $26,65^{\circ}$ que é característica da presença de quartzo e estão bem documentadas nas literaturas de (Chen, et al., 2008; Chisholm, 1992; Christ, et al., 1969; Jeffers \& Reynolds, 1987).

Perfil cristalino da amostra da atapulgita estudada apresenta uma alta cristalinidade, estando presentes praticamente atapulgita e quartzo, sendo a reflexão $\mathrm{d}_{(110)}$ da atapulgita de maior intensidade em relação à reflexão do quartzo, o que sugere uma alta pureza na amostra.

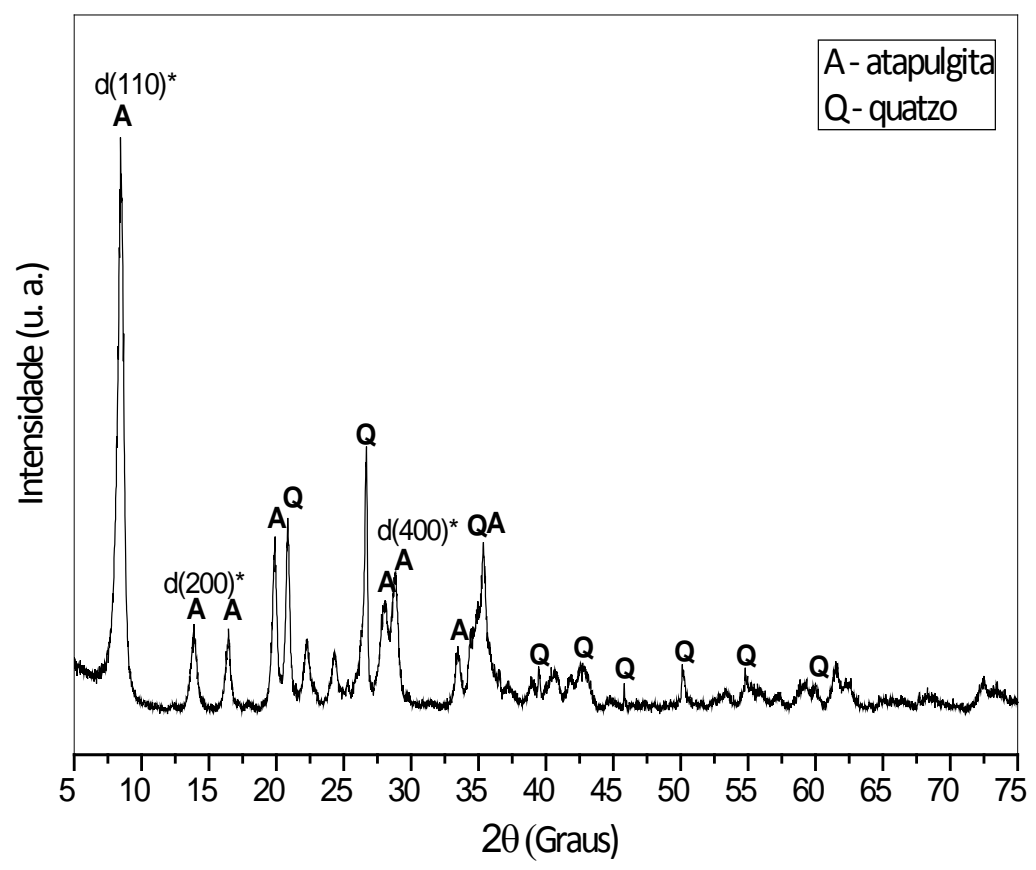

Figura 3 - Difratograma de raios- $X$ da atapulgita.

Análise de FRX

A Tabela 1 mostra a análise da composição química obtida por fluorescência de raios-X dos principais óxidos presentes na amostra de paligorsquita natural. Na composição química 
estão presentes os teores dos principais óxidos que compõe a estrutura cristalina: $\mathrm{MgO}, \mathrm{Al}_{2} \mathrm{O}_{3}$, $\mathrm{SiO}_{2}$ e $\mathrm{Fe}_{2} \mathrm{O}_{3}$ com valores iguais a 8,28\%, 11,48\% 63,67\% e 3,27\% respectivamente. A fórmula química para a meia célula unitária foi calculada pela estequiometria das composições representativas tendo como base os ânions $\mathrm{O}_{20}(\mathrm{OH})_{2} \cdot 4 \mathrm{H}_{2} \mathrm{O}$ e foi deduzida de acordo com o modelo de Bradley (1940), como $\left(\mathrm{Si}_{8,05}\right)\left(\mathrm{Mg}_{1,59} \mathrm{Al}_{1,74} \mathrm{Fe}_{0,32} \mathrm{Ti}_{0,084} \mathrm{Mn}_{0,023}\right)\left(\mathrm{K}_{0,052}\right) \mathrm{O}_{20}(\mathrm{OH})_{2}\left(\mathrm{OH}_{2}\right)_{4}$ $4 \mathrm{H}_{2} \mathrm{O}$. Está fórmula está de acordo com pesquisas desenvolvidas por (Corma, et al., 1987; Frini-Srasra \& Srasra, 2008; Gonzalez, et al., 1989b; Myriam, et al., 1998; Santos, 1975), onde o nox das espécies mais estáveis foi levado em consideração.

Tabela 1. Composição química (\%) das amostras de paligorsquita natural.

\begin{tabular}{|l|c|c|c|c|c|c|c|c|}
\hline Amostra & $\mathrm{MgO}$ & $\mathrm{Al}_{\mathbf{2}} \mathrm{O}_{\mathbf{3}}$ & $\mathrm{SiO}_{\mathbf{2}}$ & $\mathrm{K}_{\mathbf{2}} \mathrm{O}$ & $\mathrm{TiO}_{2}$ & $\mathrm{MnO}$ & $\mathrm{Fe}_{2} \mathrm{O}_{\mathbf{3}}$ & Perda ao fogo \\
\hline Atapulgita & 8,28 & 11,48 & 63,67 & 0,32 & 0,87 & 0,21 & 3,27 & 11,90 \\
\hline
\end{tabular}

Análise de FTIR

$\mathrm{Na}$ espectroscopia vibracional na região do infravermelho mostrada na Figura 2, as ligações observadas presentes na estrutura cristalina da atapulgita, são referentes aos três tipos de águas. Khorami \& Lemieux (1989) afirmam que vibrações de grupos hidroxilas coordenado ao metal e ao poro na folha octaédrica, onde os mesmos ocupam duas posições ligados a íons de $\mathrm{Mg}$ ou $\mathrm{Al}$, são chamadas de água de cristalização. Alem desta, apresenta hidroxila ligada à estrutura do metal na rede cristalina e moléculas de água contidas nos canais, sendo comumente chamada de água zeolítica.

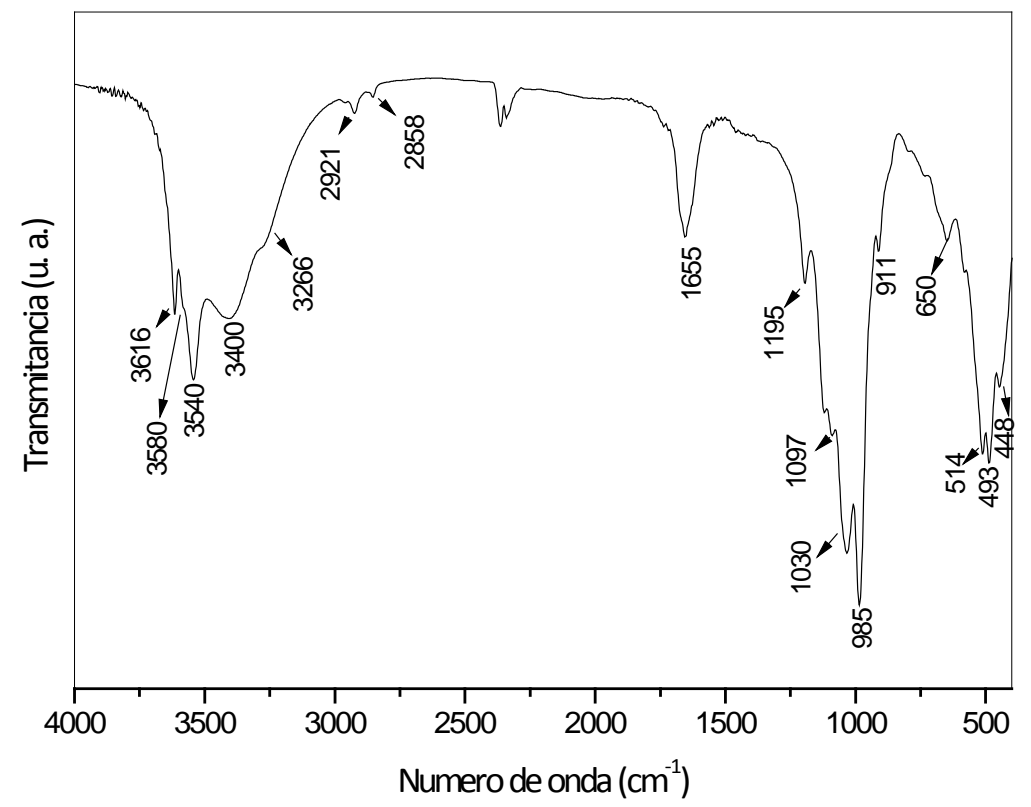

Figura 4 - FTIR da amostra de atapulgita natural.

O espectro de infravermelho da atapulgita mostra as principais bandas de vibrações características à hidroxila ligada ao metal presentes nas regiões entre 3616 a $3266 \mathrm{~cm}^{-1}$. Estas bandas, identificadas e mencionadas por Suárez \& García-Romero (2006) referem-se ao modo vibracional $2 \mathrm{Al}_{2}-\mathrm{OH}$ no octaedro. Outras bandas também presentes no espectro em 3580, 
3540,3400 e $3266 \mathrm{~cm}^{-1}$ são atribuídas a estiramento vibracional característico de $\mathrm{Al}-\mathrm{Fe}^{3+}-\mathrm{OH}$ ou Al-Mg-OH Cheng, et al. (2011). Bandas em uma região de menor número de onda presentes no espectro foram identificadas e são mostradas na Tabela 2.

Tabela 2. Bandas de vibrações no infravermelho da atapulgita.

\begin{tabular}{|c|c|c|}
\hline $\mathrm{N}^{\circ}$ de onda $\left(\mathrm{cm}^{-1}\right)$ & Bandas de atribuição & Referências \\
\hline 3616 & $\begin{array}{c}\text { Estiramento } 2 \mathrm{M}_{2}-\mathrm{OH} \\
\text { onde } \mathrm{M}=\mathrm{Al}\end{array}$ & $\begin{array}{c}\text { Khorami \& Lemieux (1989); Suárez \& } \\
\text { García-Romero (2006) }\end{array}$ \\
\hline 3580 & $\begin{array}{c}\text { Estiramento } \mathrm{Al}-\mathrm{Fe}^{3+}-\mathrm{OH} \\
\text { ou } \mathrm{Al}-\mathrm{Mg}-\mathrm{OH}\end{array}$ & Suárez \& García-Romero (2006) \\
\hline 3540 & $\begin{array}{l}\text { Estiramento } \mathrm{Al}_{2}-\mathrm{OH} \text { ou } \\
\qquad\left(\mathrm{Fe}^{3+}, \mathrm{Mg}\right)-\mathrm{OH}\end{array}$ & $\begin{array}{l}\text { Boudriche, et al. (2012); Cai, et al. } \\
\text { (2007); Cheng, et al. (2011) }\end{array}$ \\
\hline 3400 & $\begin{array}{c}\text { Estiramento de Água de } \\
\text { zeólitas (Fe }-\mathrm{Mg}-\mathrm{OH}) \text { ou } \\
(\mathrm{Al}-\mathrm{Mg}-\mathrm{OH})\end{array}$ & Suárez \& García-Romero (2006) \\
\hline 3266 & $\begin{array}{c}\text { Estiramento de Água de } \\
\text { zeólitas (Fe }-\mathrm{Mg}-\mathrm{OH}) \text { ou } \\
(\mathrm{Al}-\mathrm{Mg}-\mathrm{OH})\end{array}$ & Cheng, et al. (2011) \\
\hline 1655 & $\begin{array}{l}\text { Deformação de Água de } \\
\text { zeolíticas }\end{array}$ & Suárez \& García-Romero (2006) \\
\hline 1195 & Estiramento $\mathrm{Si}-\mathrm{O}-\mathrm{Si}$ & $\begin{array}{c}\text { Cheng, et al. (2011); Gonzalez, et al. } \\
\text { (1989b) }\end{array}$ \\
\hline 1097 & Estiramento Si - O & Cheng, et al. (2011) \\
\hline 985 & Deformação Si - OH & Cai, et al. (2007) \\
\hline 650 & Deformação $\mathrm{M}-\mathrm{OH}$ & Cai, et al. (2007) \\
\hline
\end{tabular}

Análise de MEV

A análise da MEV fornece informações sobre as microestruturas da atapulgita. A Figura 3 mostra as micrografias da amostra de Guadalupe-PI na qual exibe formas fibrosas, formando aglomerados de fitas e agulhas com comprimentos variados Gan, et al. (2009).

O histograma da Figura 3 é referente ao estudo mais detalhado do comprimento médio das fibras da atapulgita de Guadalupe-PI medidos a partir das imagens da microscopia, no qual foram analisadas 50 partículas do argilomineral e realizada a contagem. De acordo com as medidas, o comprimento médio da fibra varia de 0,3 a 1 micrômetro, mas com a predominância do comprimento de $1 \mu \mathrm{m}$. 


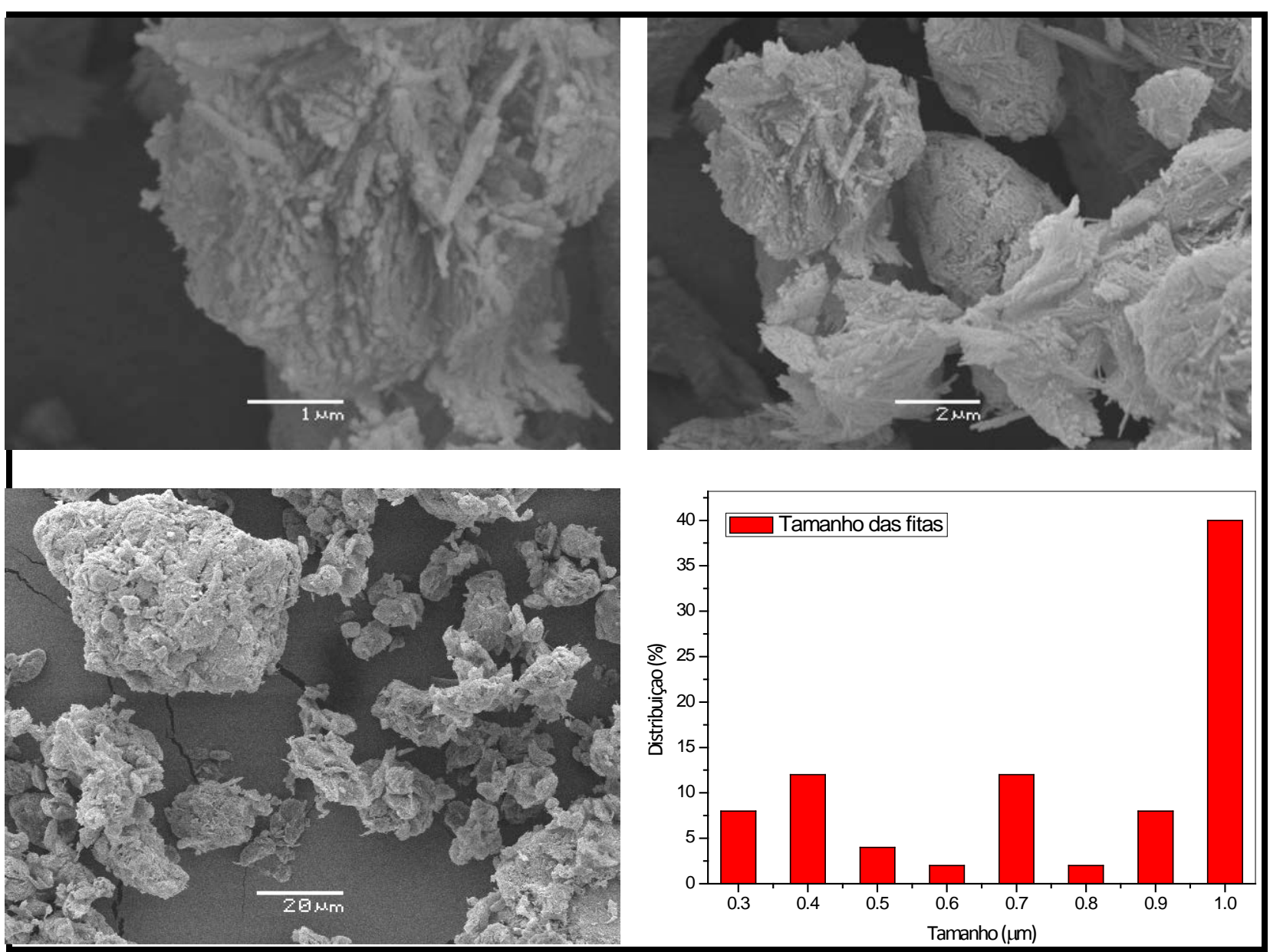

Figura 5 - Microscopias da atapulgita natural (1, 2 e $20 \mu \mathrm{m})$ e tamanho médio do comprimento da fibra.

\section{Análises de BET e CTC}

A área superficial na amostra atapulgita natural apresentou um valor relativamente alto de $113 \mathrm{~m}^{2} \cdot \mathrm{g}^{-1}$ conforme exposto na tabela 3. Este valor quando comparado com os trabalhos da literatura utilizando atapulgita de varias regiões do mundo, o mesmo encontrase dentro de um valor intermediário.

A análise de capacidade de troca catiônica da atapulgita indicou que esta apresenta um excelente valor de troca com $40 \mathrm{meq} / 100 \mathrm{~g}$ e este resultado se encontram dentro do valor de CTC esperado quando comparando com os dados da literatura (Chen \& Wang, 2007; Chen, et al., 2011; Gonzalez, et al., 1989a) 
Tabela 3. Resultado da àrea superficial especifica da atapulgita.

\begin{tabular}{|l|c|c|}
\hline Amostras & BET $\left(\mathrm{m}^{2} \cdot \mathrm{g}^{-1}\right)$ & Referências \\
\hline Segovia (Spanish) & 138 & Gonzalez, et al. (1989a) \\
\hline Xuyi County (China) & 206 & Ye, et al. (2006) \\
\hline Gansu (China) & 48.66 & Chen \& Wang (2007) \\
\hline Tunisia & 67.4 & Frini-Srasra \& Srasra (2008) \\
\hline Xuyi County (China) & 51.3 & Gan, et al. (2009) \\
\hline Jiangsu, (China) & 123 & Zhang, et al. (2010) \\
\hline Região Ghoufi (Algeria) & 125.1 & Boudriche, et al. (2011) \\
\hline Guadalupe -PI & 113 & Este trabalho \\
\hline
\end{tabular}

\section{CONCLUSÕES}

Os resultados das técnicas utilizadas na caracterização da atapulgita de Guadalupe-PI mostraram que este argilomineral apresenta uma grande cristalinidade com a reflexão $d_{(110)}$ referente à atapulgita com maior intensidade em relação a do quartzo, conforme mostrou a análise de DRX. Na composição química da atapulgita estão presentes os principais óxidos que constituem a estrutura cristalina, de acordo com a literatura especializada. A análise de infravermelho mostrou que as principais bandas presentes na estrutura da atapulgita são referentes aos grupos hidroxilas coordenados ao metal na folha octaédrica e águas zeolíticas. As micrografias mostraram que este argilomineral apresenta uma morfologia fibrosa com tamanho de fibras variando de 0,3 a 1,0 $\mu \mathrm{m}$, mas com a predominância do tamanho de fitas de 1,0 $\mu \mathrm{m}$. A análise de área superficial especifica indicou que o argilomineral em estudo possui um tamanho de área de $113 \mathrm{~m}^{2} / \mathrm{g}$ e valor de troca catiônica de $40 \mathrm{meq} / 100 \mathrm{~g}$. Estes valores mostraram que a argila atapulgita de Guadalupe-PI possui um grande potencial tecnológico para aplicação industrial.

\section{AGRADECIMENTOS}

Os autores do trabalho agradecem ao Centro de Tecnologia Mineral - CETEM, pela análise de FRX, e a empresa Itaoeste pelo fornecimento da amostra de argila e pelas análises de MEV e BET e a Universidade Federal do Piauí - UFPI.

\section{REFERÊNCIAS BIBLIOGRÁFICAS}

1. A. MIFSUD,M. RAUTUREAU,FORNES, A.V.; Etude de l'eau dans la palygorskite a l'aide des analyses thermiques Clay Minerals, v. 13, n. p. 367-374, 1978. 
2. ÁLVAREZ-AYUSO, E.,GARCÍA-SÁNCHEZ, A.; Removal of cadmium from aqueous solutions by palygorskite. Journal of Hazardous materials, v. 147, n. 1-2, p. 594-600, 2007.

3. BALTAR, C.A.M.,DA LUZ, A.B.,BALTAR, L.M.,DE OLIVEIRA, C.H.,BEZERRA, F.J.; Influence of morphology and surface charge on the suitability of palygorskite as drilling fluid. Applied Clay Science, v. 42, n. 3-4, p. 597-600, 2009.

4. BOUDRICHE, L.,CALVET, R.,HAMDI, B.,BALARD, H.; Effect of acid treatment on surface properties evolution of attapulgite clay: An application of inverse gas chromatography. Colloids and Surfaces A: Physicochemical and Engineering Aspects, v. 392, n. 1, p. 45-54, 2011.

5. BOUDRICHE, L.,CALVET, R.,HAMDI, B.,BALARD, H.; Surface properties evolution of attapulgite by IGC analysis as a function of thermal treatment. Colloids and Surfaces, A: Physicochemical and Engineering Aspects, v. 399, n. 0, p. 1-10, 2012.

6. CAI, Y.,XUE, J.,POLYA, D.A.; A Fourier transform infrared spectroscopic study of Mg-rich, Mg-poor and acid leached palygorskites. Spectrochim. Acta, Part A v. 66, n. 2, p. 282-288, 2007.

7. CHEN, H.,WANG, A.; Kinetic and isothermal studies of lead ion adsorption onto palygorskite clay. Journal of Colloid and Interface Science, v. 307, n. 2, p. 309-316, 2007.

8. CHEN, H.,ZHAO, J.,ZHONG, A.G.,JIN, Y.X.; Removal capacity and adsorption mechanism of heattreated palygorskite clay for methylene blue. Chemical Engineering Journal, v. 174, n. 1, p. 143150, Oct. 2011.

9. CHEN, T.,WANG, H.J.,ZHANG, X.P.,ZHENG, N.; SAED and HRTEM investigation of palygorskite. Acta Geologica Sinica-English Edition, v. 82, n. 2, p. 385-391, Apr. 2008.

10. CHENG, H.,YANG, J.,FROST, R.L.,WU, Z.; Infrared transmission and emission spectroscopic study of selected Chinese palygorskites. Spectrochimica Acta, Part A: Molecular and Biomolecular Spectroscopy, v. 83, n. 1, p. 518-524, 2011.

11. CHISHOLM, J.E.; Powder-diffraction patterns and structural models for palygorskite. Canadian Mineralogist, v. 30, n. p. 61-73, Mar. 1992.

12. CHRIST, C.L.,HATHAWAY, J.C.,HOSTETLE.PB,SHEPARD, A.O.; Palygorskite-new X-ray data. American Mineralogist, v. 54, n. 1-2, p. 198-\&, 1969.

13. CORMA, A.,MIFSUD, A.,SANZ, E.; Influence of the chemical - composition and textural characteristics of palygorskite on the acid leaching of octahedral cations. Clay Minerals, v. 22, n. 2, p. 225-232, Jun. 1987.

14. FRINI-SRASRA, N.,SRASRA, E.; Effect of heating on palygorskite and acid treated palygorskite properties. Surface Engineering and Applied Electrochemistry, v. 44, n. 1, p. 43-49, 2008.

15. GAN, F.Q.,ZHOU, J.M.,WANG, H.Y.,DU, C.W.,CHEN, X.Q.; Removal of phosphate from aqueous solution by thermally treated natural palygorskite. Water Research, v. 43, n. 11, p. 2907-2915, Jun. 2009.

16. GARCIA-ROMERO, E.,BARRIOS, M.S.,REVUELTA, M.A.B.; Characteristics of a Mg-palygorskite in miocene rocks, Madrid Basin (Spain). Clays and Clay Minerals, v. 52, n. 4, p. 484-494, Aug. 2004.

17. GIONIS, V.,KACANDES, G.H.,KASTRITIS, I.D.,CHRYSSIKOS, G.D.; On the structure of palygorskite by mid- and near-infrared spectroscopy. American Mineralogist, v. 91, n. 7, p. 1125-1133, Jul. 2006.

18. GONZALEZ, F.,PESQUERA, C.,BENITO, I.,MENDIOROZ, S.,PAJARES, J.A.; Mechanism of acid activation of magnesic palygorskite. Clays and Clay Minerals, v. 37, n. 3, p. 258-262, Jun. 1989a.

19. GONZALEZ, F.,PESQUERA, C.,BLANCO, C.,BENITO, I.,MENDIOROZ, S.,PAJARES, J.A.; Structural and textural evolution of Al- and Mg-rich palygorskites, I. Under acid treatment. Applied Clay Science, v. 4, n. 4, p. 373-388, 1989b.

20. JEFFERS, J.D.,REYNOLDS, R.C.; Expandable palygorskite from the cretaceous-tertiary, mangyshlak peninsula, USSR. Clays and Clay Minerals, v. 35, n. 6, p. 473-476, Dec. 1987.

21. KHORAMI, J.,LEMIEUX, A.; Comparison of attapulgites from different sources using TG/DTG and FTIR. Thermochimica Acta, v. 138, n. 1, p. 97-105, 1989.

22. LEI, Z.Q.,WEN, S.X.; Synthesis and decoloration capacity of well-defined and WMA-grafted palygorskite nanocomposites. European Polymer Journal, v. 44, n. 9, p. 2845-2849, Sep. 2008. 
23. $\mathrm{LI}, \mathrm{X} ., \mathrm{NI}, \mathrm{C} ., \mathrm{YAO}, \mathrm{C} ., \mathrm{CHEN}, \mathrm{Z}$; Development of attapulgite/Ce1-xZrxO2 nanocomposite as catalyst for the degradation of methylene blue. Applied Catalysis B: Environmental, v. 117-118, n. 0, p. 118-124, 2012.

24. LIU, Y.F.,HUANG, J.H.,WANG, X.G.; Adsorption isotherms for bleaching soybean oil with activated attapulgite. Journal of the American Oil Chemists Society, v. 85, n. 10, p. 979-984, Oct. 2008.

25. LUZ, A.B.,ALMEIDA, S.L.M. Cap. 9. Argila/Atapulgita e Sepiolita. Rochas \& Minerais Industriais: Usos e Especificações. 2a Edição revisada e ampliada. 2005. ISBN 85-7227-204-6.

26. LUZ, A.B.,ALMEIDA, S.L.M.,RAMOS, L.T.S. Estudos tecnológicos para o aproveitamento da atapulgita de Guadalupe-PI. Série Tecnologia Mineral. $n^{\circ} 42.1988$.

27. MORALES-CARRERA, A.M.,VARAJÃO, A.F.D.C.,GONÇALVES, M.A.,STACHISSINI, A.S.; Argilas bentoníticas da península de Santa Elena, Equador: pilarização, ativação ácida e seu uso como descolorante de óleo de soja. Química Nova, v. 32, n. p. 2287-2293, 2009.

28. MYRIAM, M.,SUAREZ, M.,MARTIN-POZAS, J.M.; Structural and textural modifications of palygorskite and sepiolite under acid treatment. Clays and Clay Minerals, v. 46, n. 3, p. 225-231, 1998.

29. NEAMAN, A.,SINGER, A.; The effects of palygorskite on chemical and physico-chemical properties of soils: a review. Geoderma, v. 123, n. 3-4, p. 297-303, 2004.

30. NETO, J.P.,ALMEIDA, S.L.M.,CARVALHO, R.D.M. Atapulgita do Piauí para industria farmacêutica. Tecnologia Mineral. $n^{\circ}$ 64. CETEM/CNPq, 1993. ISBN 85-7227-040X.

31. SANTOS, P.D.S. Tecnologia de argilas. Ed. da USP/Edcard Blucher LTDA: São Paulo. 1975.

32. STATHATOS, E.,PAPOULIS, D.,AGGELOPOULOS, C.A.,PANAGIOTARAS, D.,NIKOLOPOULOU, A.; TiO2/palygorskite composite nanocrystalline films prepared by surfactant templating route: Synergistic effect to the photocatalytic degradation of an azo-dye in water. Journal of Hazardous materials, v. 211-212, n. 0, p. 68-76, 2012.

33. SUÁREZ, M.,GARCÍA-ROMERO, E.; FTIR spectroscopic study of palygorskite: Influence of the composition of the octahedral sheet. Applied Clay Science, v. 31, n. 1-2, p. 154-163, 2006.

34. WANG, K.,WANG, H.,PASUPATHI, S.,LINKOV, V.,JI, S.,WANG, R.; Palygorskite promoted PtSn/carbon catalysts and their intrinsic catalytic activity for ethanol oxidation. Electrochimica Acta, v. 70, n. 0 , p. 394-401, 2012.

35. YE, H.,CHEN, F.,SHENG, Y.,SHENG, G.,FU, J.; Adsorption of phosphate from aqueous solution onto modified palygorskites. Separation and Purification Technology, v. 50, n. 3, p. 283-290, 2006.

36. ZHANG, J.,WANG, Q.,CHEN, H.,WANG, A.; XRF and nitrogen adsorption studies of acid-activated palygorskite. Clay Minerals, v. 45, n. 2, p. 145-156, 2010. 